\title{
Scalable All-Printed Microwave Microfluidic Sensor for Multi- Liquid Characterization based on a Stub-Loaded Microstrip Line
}

This paper was downloaded from TechRxiv (https://www.techrxiv.org).

LICENSE

CC BY 4.0

SUBMISSION DATE / POSTED DATE

28-07-2021 / 02-08-2021

\section{CITATION}

Wagih, Mahmoud; Shi, Junjie (2021): Scalable All-Printed Microwave Microfluidic Sensor for Multi-Liquid Characterization based on a Stub-Loaded Microstrip Line. TechRxiv. Preprint. https://doi.org/10.36227/techrxiv.15067551.v1

$\mathrm{DOI}$

10.36227/techrxiv.15067551.v1 


\title{
Scalable All-Printed Microwave Microfluidic Sensor for Multi-Liquid Characterization based on a Stub-Loaded Microstrip Line
}

\author{
Mahmoud Wagih, Member, IEEE, and Junjie Shi
}

\begin{abstract}
Microwave microfluidic sensors are typically designed with a channel in vicinity of a resonator's fringing electric $(E)$-fields, to characterize the material properties of a single fluid. This paper leverages hybrid 3D and dispenser printing to realize a scalable microfluidic sensor utilizing the parallelplate capacitance of an open-ended microstrip stub, enabling, for the first time, a tunable sensitivity. A stub-loaded microstrip line is then proposed for characterizing multiple microfluidic samples simultaneously using a simple two-port multi-band resonator. The physical constrains which limit the scalability of the proposed sensors have been analyzed analytically and numerically, prior to implementing a three-channel triple-band sensor. The microfluidic channels have been fabricated using stereolithography 3D printing with the microstrip line directly dispenser printed on a conformable polyimide substrate. To accommodate varying channel thicknesses, a tapered microstrip line is proposed to maintain the impedance matching. The fabricated sensor is characterized using binary water-IPA mixtures to evaluate its sensitivity, comparing favorably with reported 3D-printed sensors. The proposed sensor achieves over $90 \%$ accuracy in determining the real permittivity following a simple water-based calibration across the different channels, for samples with $16<\epsilon_{r}<78$. In addition to achieving a sensitivity in-line with state-of-the-art single-channel printed sensors, the sensor's applicability to temperature sensing is demonstrated experimentally with over $2.4-5 \mathrm{MHz} /{ }^{\circ} \mathrm{C}$ temperature sensitivity across all channels.
\end{abstract}

Index Terms-Antennas, Filter, Microfluidic, Microstrip, Resonant Sensors, Sensor,

\section{INTRODUCTION}

$\mathbf{M}$ ICROWAVE resonators have recently attracted significant interest for a plethora of sensing applications [1] [3]. From chipless wireless RFID "smart" sensing [1], to onchip microwave, mmWave [4], and $\mathrm{THz}$ material characterization [5], microwave sensing covers a range of industrial to healthcare applications [6], [7]. Compared to chemical methods, microwave sensors are non-destructive, non-invasive, and can sense various stimulants such as gas concentrations [8], glucose levels [7], and bacterial growth in real-time [6].

Resonant dielectric sensors are preferred over broadband phase-delay sensors for applications where a high sensitivity is required for small changes in the relative permittivity of a

This work was supported by the UK Engineering and Physical Sciences Research Council (EPSRC) under Grant EP/P010164/1. (Corresponding author: Mahmoud Wagih)

The authors are with the School of Electronics and Computer science, University of Southampton, Southampton, SO17 1BJ, U.K. (email:mahm1g15@ecs.soton.ac.uk)

Digital Object Identifier: , datasets used in this paper will be made available from the University of Southampton repository at DOI: X microfluidic Material under Test (MUT) [9]. Planar resonators such as split ring resonators (SRRs) [7], [10], [11], series metamaterial-like LC resonator [12], interdigitated capacitors within an SRR [9], spoof surface plasmons [13] and more recently susbtrate integrated waveguide (SIW) cavity [14] resonators have been widely investigated for microfluidic and material sensing. The quality factor and the capacitance or inductance of the sensor are often discussed when improving the sensitivity of such sensors [13]. However, most planar sensors are based on a microfluidic channel placed on top of or going through the planar resonator, [7], [9], [10], [13], [15], utilizing the fringing electric $(E)$-fields going through the MUT for sensing. Moreover, while certain resonator designs may improve the sensitivity based on a high Q-factor, designing a sensor based on fringing $E$-fields will only yield minimal improvements to the sensitivity due to the existence of multiple capacitive elements, not contributing to sensing [16]. Therefore, the use of the parallel plate capacitance as opposed to the fringing $E$-fields was proposed for improving the sensitivity compared to conventional planar resonators [16].

Additive manufacturing, using direct-write 3D and conductor printing, has been widely used to realize microwave and mmWave components such as filters, antennas, and transmission lines [?], [14], [15], [17]-[23]. Recently, additive manufacturing using fused deposition modelling (FDM) has been proposed a method of realizing microfluidic channels for integration with resonators implemented on a conventional printed circuit board (PCB) [15]. To this end, the resonator and the channel are designed as separate entities, which are then integrated after fabrication This restricts the MUT to being deployed over a planar microstrip resonator, typically existing in its fringing field region [15]. However, utilizing the fringing capacitance of a complex structure such as a split ring resonator reduces the overall sensitivity of the sensor due to the existence of several capacitive elements in the equivalent circuit of the sensing resonator [16]. Therefore, the use of the parallel-plate capacitance of a microstrip was proposed for high-sensitivity sensing [24]. However, the frugal realization of the sensor limits its scalability [16]. 3D printing was used to realize a microfluidic channel burried beneath a microstrip line [22]. The use of stereolithography (SLA) printing to realize a buried meander-line microfluidic channel, buried within a substrate-integrated waveguide (SIW) resonant cavity, was also proposed [14]. While these implementations shows the flexibility of 3D printed channels and their improved integration 
(a)

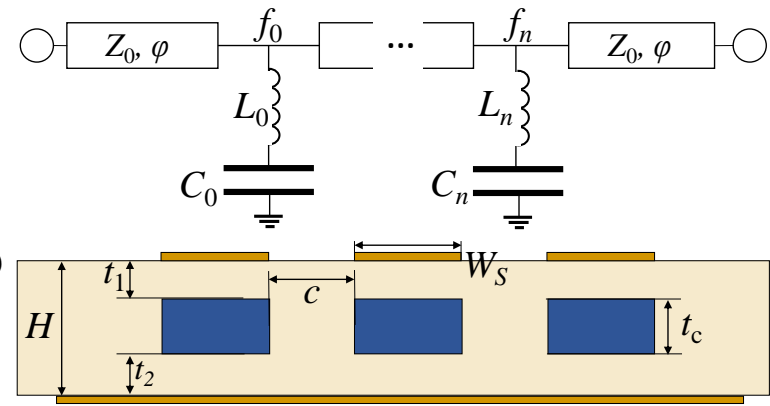

Fig. 1. Equivalent circuit of the stub-loaded dielectric sensing microstrip line (a) and the cross-section of a triple-channel/stub microstrip sensor (b).

with microstrip structures, the ability to 3D print the channel was not applied to improving the sensor's sensitivity, or to enable scalable multi-parameter sensors.

This paper proposes a stub-loaded microstrip line for simultaneously characterizing multiple liquid dielectric samples, representing the first scalable microwave sensor design capable of characterizing more than two samples. In Section II, microstrip stubs are investigated from a dielectric sensing perspective, followed by closed-form circuit analysis and fullwave simulations. Section III discusses the fabrication of the sensor using low-cost 3D substrate and dispenser microstrip printing along with the measured results. Finally, the dielectric extraction, sensitivity analysis and sensor accuracy evaluated are presented in Section IV, along with a qualitative and quantitative comparison with state-of-the-art sensors.

\section{Microstrip Sensor Design And Simulation}

\section{A. Parallel-Plate Resonant Microstrip Sensor Design}

A parallel plate microstrip capacitor exhibits a significantly higher $E$-field density over a larger area, compared to the fringing fields of a coupled resonator. While an open-ended microstrip stub was used in [25] for sensing applications, the MUT was placed above the parallel line, implemented on a standard PCB, resulting in a standard fringing capacitance sensor similar to [26]. 3D printing was previously used to implement a channel buried underneath a microstrip line on a standard PCB [22].

With the MUT buried in the microstrip substrate, as shown in Fig. 1(b), the microfluidic channel can now be regarded as a multi-layered substrate [27]. The equivalent circuit of the dielectric sensor can then be simplified to a single LC element. The capacitive element is dependent on the MUT's permittivity $\left(\epsilon_{\mathrm{MUT}}\right)$. However, for the multi-material susbtrate, the equivalent real permittivity, $\epsilon_{\mathrm{Eq}}$. is given by

$$
\epsilon_{\mathrm{Eq} .}=\sum_{n=1}^{N}\left(\frac{t_{n}}{\epsilon_{n}}\right)^{-1} \times H
$$

where $H$ is the total height of the substrate, $t_{n}$ is the thickness of the individual layers from Fig. 1, and $\epsilon_{n}$ is the permittivity of layer $n$ [27].

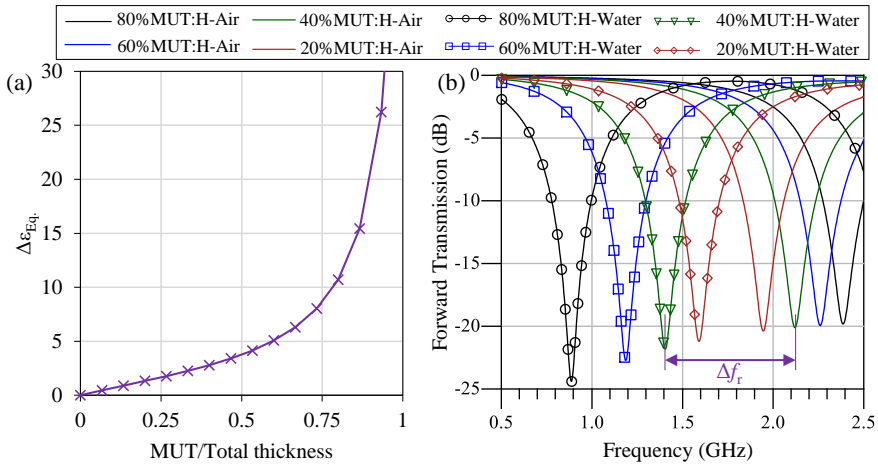

Fig. 2. Analytically calculated $\Delta \epsilon_{\mathrm{Eq}}$. as the a function of the MUT-to-channel thickness (a), and the influence in the sensor's $\Delta f_{r}$ sensitivity.

As varying the MUT varies $\epsilon_{\mathrm{MUT}}$ and hence $\epsilon_{\mathrm{Eq} \text {., }}$, the maximum change in the resonant frequency $\Delta f_{r}$ for a sensing microstrip stub can be given by the

$$
\Delta f_{r}=\frac{1}{2 \pi \sqrt{L \Delta C}},
$$

where $L$ represents the inductance of the resonant microstrip stub, and $\Delta C_{n}$ represents the change in the capacitance induced by the MUT [16]. The sensitivity $S$ of a resonant dielectric sensor is in-turn given by

$$
S=\frac{f_{\text {air }}-f_{\epsilon_{\mathrm{r}}}}{f_{\text {air }}\left(\epsilon_{\mathrm{r}}-1\right)},
$$

where $f_{\text {air }}$ and $f_{\epsilon_{\mathrm{r}}}$ are the resonant frequencies in air and for an MUT-loaded sensor [14].

Typically, maximizing $S$ is regarded as a figure of merit in dielectric sensor design [16], [28]. Observing (1) and (2), it can be seen that varying $\epsilon_{\text {Eq. }}$, through controlling the height of the substrate and the channel, can increase the variation in capacitance and hence, enhancing $S$.

To demonstrate the effect of varying thicknesses on $S, H=3$ $\mathrm{mm}$ is considered with varying $t_{c}$ to $t_{1}$ and $t_{2}$ ratios, in the case of a single resonant microstrip stub. MUT $\epsilon_{r}$ of 78 and 1.0, representative of water and air, are assumed for the loaded and unloaded cases. $\epsilon_{\mathrm{Eq}}$. has been calculated analytically using (1), for varying $t_{c} / H$ ratios. A substrate $\epsilon_{r}=2.7$ is assumed in this calculation. Fig. 2(a) shows the analytically-calculated variation in $\epsilon_{\mathrm{Eq}}$. as a function of the channel-to-substrate thickness, where it can be observed that orders of magnitude change in $\epsilon_{\text {Eq. }}$. could be introduced to the sensor by controlling the channel's height.

Fig. 2(b) shows the closed-form simulated forward transmission response of a microstrip stub with length $L=30 \mathrm{~mm}$, simulated using Keysight ADS, for varying MUT-to-substrate height ratios. It can be clearly observed that the change in the resonance frequency $\Delta f_{r}$ is highly dependent on the channel's height, where the sensitivity can be improved by up to four-folds, by changing the MUT-to-substrate ratio from $20 \%$ to $80 \%$. Therefore, by combining the simple parallel-plate capacitance of a microstrip resonator with high-resolution additive manufacturing, microwave microfluidic sensors with high tunable sensitivities can be realized. 


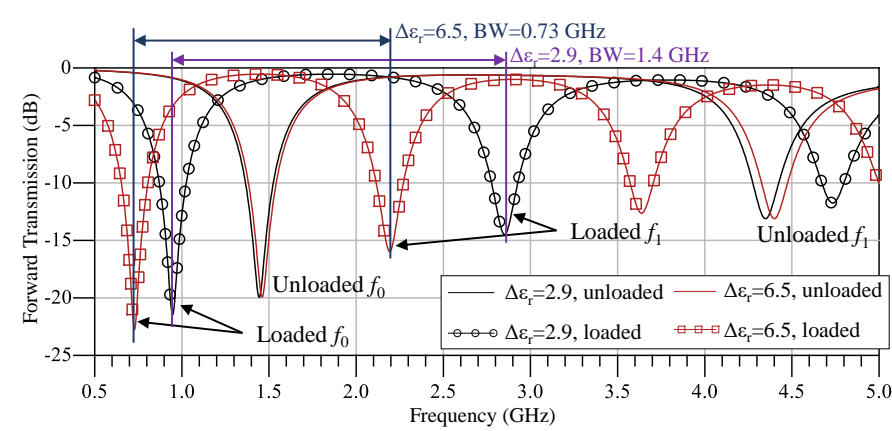

Fig. 3. Broadband forward transmission of two microstrip sensors with different $\Delta \epsilon_{r}$, showing their usable scaling bandwidth (BW) for multimaterial characterization.

\section{B. Multi-Fluid Sensor Design}

So far, only a single sensing element has been discussed. As shown in Fig. 1, a microstrip line could be loaded with multiple sensing elements, i.e. resonant stubs, for simultaneously characterizing multiple fluids using the same read-out circuit, such as an oscillator [29], significantly reducing the testing time, connections, and cost. The limit of such sensor scaling mechanism is the bandwidth. To explain, the openended line resonates at at $L=\lambda / 4$ and $3 \lambda / 4$. Therefore, should additional sensing elements be added to the line, their resonant frequency has to fall between the first and secondorder resonances of the lowest-frequency stub.

A numerical example is considered to illustrate the bandwidth limits of a multi-stub sensor. Stub $L=44 \mathrm{~mm}$, substrate $H=1.44 \mathrm{~mm}$, and $W_{S}=1.4 \mathrm{~mm}$ are assumed. Unloaded $\epsilon_{\mathrm{Eq} .}=1.6$ and 1.55 are investigated for loaded $\Delta \epsilon_{\mathrm{Eq} .}=2.9$ and 6.5 , respectively. The microstrip stubs' broadband $S_{21}$ has been simulated for both the loaded and unloaded cases, using the closed-form model, and is shown in Fig. 3.

Observing Fig. 3, the trade-off between the sensitivity and multi-material scalability becomes apparent. To explain, while a higher $\Delta \epsilon_{\text {Eq. }}$ improves the sensitivity of the microstrip resonator, resulting in a greater $\Delta f_{r}$ as previously observed in Fig. 2, it reduces the usable bandwidth between the first and second-order mode resonances. The larger change in $f_{r}$ also shifts the higher-order resonance, which reduces the higher bandwidth which can be used to add more stubs to the line for simultaneous multi-liquid characterization. To illustrate, the stub whose $\Delta \epsilon_{\mathrm{Eq}}=2.9$ achieves approximately two-folds wider bandwidth between its unloaded first-order $f_{r}$ and the second-order loaded $f_{r}$, as shown in Fig. 3.

Consequently, to enable multi-stub/multi-fluid sensors to be realized, the sensitivity could be dynamically tuned by varying the MUT-to-substrate thickness ratio. The maximum number $N$ of stubs which can be realized in the scalable bandwidth of a resonant microstrip sensor is hence given by

$$
N=\frac{3 f_{0, \mathrm{MUT}}-f_{0, \epsilon_{0}}}{\Delta f_{n}},
$$

where $\Delta f_{n}$ is the sensing bandwidth of the $n^{\text {th }}$ stub, $f_{0, \text { MUT }}$ is the resonant frequency with the maxmum MUT $\epsilon_{r}$, and $f_{0, \epsilon_{0}}$ is the unloaded resonance. Thus, as long as the sensing bandwidth of each resonant stub does not overlap the other

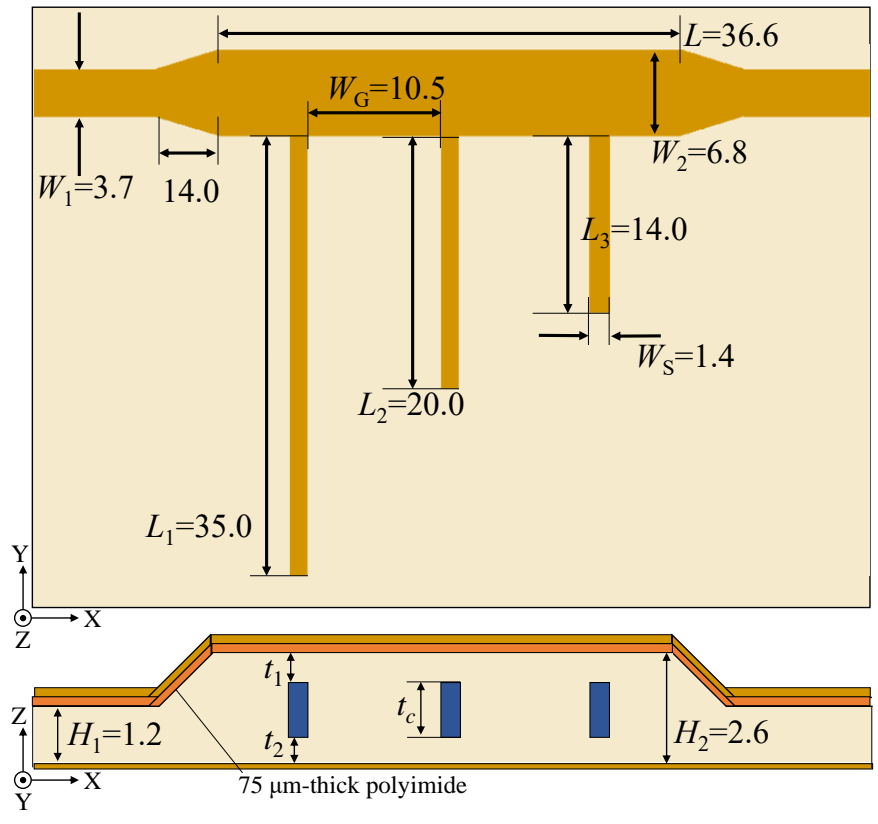

Fig. 4. Layout and dimensions of the fabricated sensor.

elements on the same two-port network, a scalable multi-fluid sensor can be realized based on stub-loaded microstrip line. However, this assumes that closely-spaced sensing elements do not interact or mutually-couple, this is investigated using 3D full-wave simulations, detailed in the next sub-section.

\section{Sensor Design and Full-Wave Simulation}

For the sensor to be fabricated using low-cost standard SLA 3D printing, the total substrate height should be over $2 \mathrm{~mm}$ [14]. Due to the high thickness, standard edge PCB RF connectors cannot be directly mounted on the substrate. To overcome the thickness issue, a ramped and tapered nonuniform microstrip line is proposed. Tapered non-uniform microstrip lines first emerged for MMIC applications [30], [31], and were more recently applied to 3D mmWave multi-layered packaging [32], enabled by the advances in 3D printing. The width and taper rate are optimized to maintain $Z_{0}=50 \Omega$ throughout the structure. The layout and dimensions of the fabricated prototype are shown in Fig. 4

So far, the closed-form microstrip circuit model has been used to simulate the sensor. However, such a model assumes the stubs are fully isolated and have no influence on each other's $f_{n}$. 3D Full-wave simulation in CST Microwave Studio was used to observe the sensor's response for MUT $\epsilon_{r}=1.0$ and 78 , representing the two ends of the possible MUTs based on recently reported sensors [28]. The first step in designing the multi-stub sensor is to identify the minimum distance required between the adjacent stubs to mitigate coupling.

The sensor has been simulated in CST Microwave Studio for varying separation, $W_{G}$, between the stubs. It was found that, based on a substrate's $\epsilon_{r}=2.7$, the minimum $W_{G}$ required to minimize the coupling between the three separate stubs and $f_{r}$ is $10.5 \mathrm{~mm}, \approx 10 \times W_{S}$, as shown in Fig. 4. The simulated response of the three resonators/channels for varying MUT $\epsilon_{r}$ 

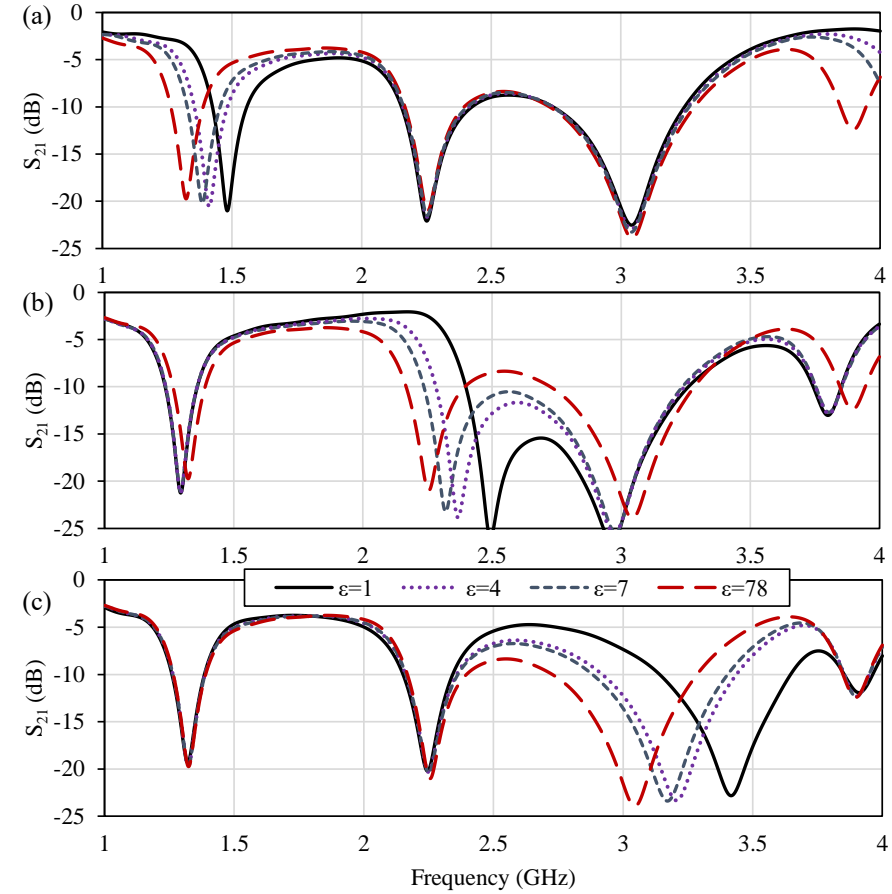

Fig. 5. Simulated $S_{21}$ response of the proposed sensor for varying- $\epsilon_{r}$ across the three channels: (a) channel 1; (b) channel 2; (c) channel 3.

is shown in Fig. 5. For each sweep, the other channels were simulated with a $\epsilon_{r}=78$ filling, representative of active operation with a water-based MUT, which emulates various real applications such as glucose monitoring [7]. Observing Fig. 5, changing $\epsilon_{r}$ in each channel introduces minimal variations in the other channels, with the most obvious change in the case of the MUT in channel 2, in Fig. 5(b), influencing $f_{1}$ and $f_{3}$ when filled with water. Such additional interference could be calibrated using a sample of known $\epsilon_{r}$, e.g. water, which is widely used to calibrate microwave permittivity sensors [33].

\section{Sensor Fabrication And Measurement}

\section{A. Hybrid Additive Manufacturing}

To fabricate the sensor with the dimensions in Fig. 4, a hybrid additive manufacturing approach is utilized. First, the substrate with an embedded channel is $3 \mathrm{D}$ printed. The microstrip line is then dispenser printed (thick-film direct-write printing technique [23], [34]) on a conformable thin substrate before integration with the channel. Similar approaches have been proposed combining inkjet printing with $3 \mathrm{D}$ printing for antenna and system-on-package applications [32]. Dispenser printing on the other hand is more suited to rapid manufacturing and prototyping compared to inkjet printing, in addition to being able to realize layers of higher thickness (and hence conductivity) in a single print, significantly reducing fabrication time and cost [23]. Fig. 6 shows the fabrication process of the sensor.

Printing the substrate represents a more straightforward and lower cost fabrication method compared to molded PDMS microfluidic channels integrated with low-loss RF PCB substrates [35]. SLA 3D printing has been selected as opposed to the

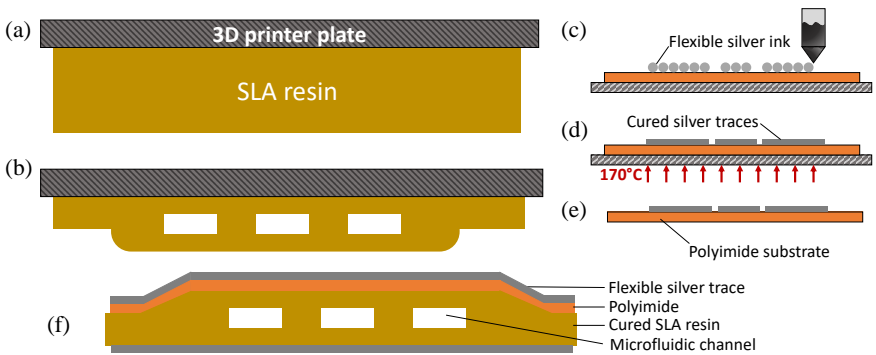

Fig. 6. Fabrication steps of the proposed sensor: (a) SLA resin prior to 3D etching; (b) 3D printed microfluidic channel resin; (c) dispenser printing of the microstrip line on a polyimide film; (d) thermal curing of the silver traces; (e) the cured conformable microstrip film; (f) the assembled 3D/dispenser printed sensor.

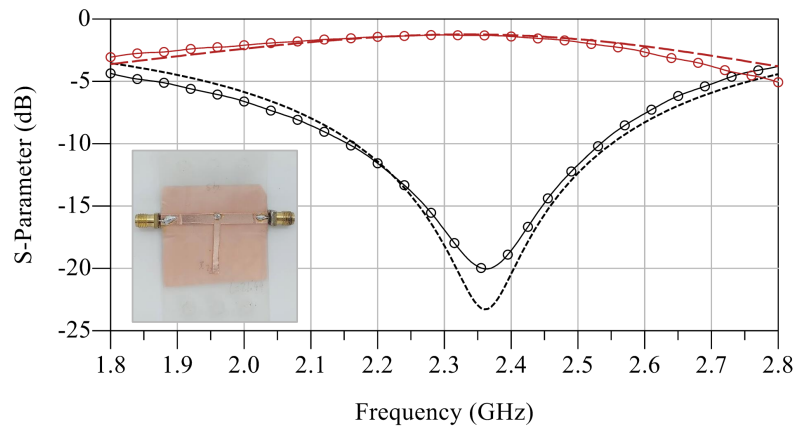

Fig. 7. Simulated and measured $S_{21}$ (black) and $S_{11}$ (red) response of a T-resonator for characterizing the 3D-printed resin's permittivity; $\epsilon_{r}=2.7$ and $\tan \delta=0.02$.

more conventional FDM, where an SLA-printed substrate will exhibit water-tight properties, preventing the substrate from absorbing the MUT. Moreover, the optical translucency of the resin (Formlabs Clear Resin) enables visualization of the MUT as it fills the channel [14]. The substrate was printed by 3D Hubs using a Formlabs SLA printer with ultraviolet (UV) postcuring. Owing to the increased thickness of the channel and the proposed ramped microstrip line, the designed sensor can be printed using low-cost low-resolution printers due to the large layer thickness of $0.7 \mathrm{~mm}$ used in this work.

A $1.6 \mathrm{~mm}$-thick SLA substrate was printed for measuring the relative permittivity of the substrate, to guide the design of the microstrip sensor. Fig. 7 shows the closed-form (ADS) simulated and measured $S_{21}$ response of the T-resonator, for $\epsilon_{r}=2.7$ and $\tan \delta=0.02$. The dimensions of the line, namely $W_{1}$ and $W_{2}$ from Fig. 4 , were selected to maintain $Z_{0}=50 \Omega$. $W_{\mathrm{S}}$ was chosen to achieve a high- $Z_{0}$ resulting in a more narrowband response to improve the resolution of the sensor.

Direct-write dispenser printing is used to fabricate the microstrip line and stub using conductive silver ink. The ink (Voltera flexible ink) was printed on a $75 \mu \mathrm{m}$ flexible polyimide substrate $\left(\epsilon_{r}=3.2, \tan \delta=0.002\right)$ using a Voltera V-One printer. Dispenser printing has previously been used to design and prototype antennas and rectennas [23], [34], showing minimal variations compared to etched copper sheets of the same geometry [34]. Following the conductor curing at $170^{\circ}$ on a standard hot-plate, the flexible microstrip line is adhered to the ramped substrate using a double-sided adhesive, of a $<50 \mu \mathrm{m}$ measured thickness. Photorgaphs and micrographs 


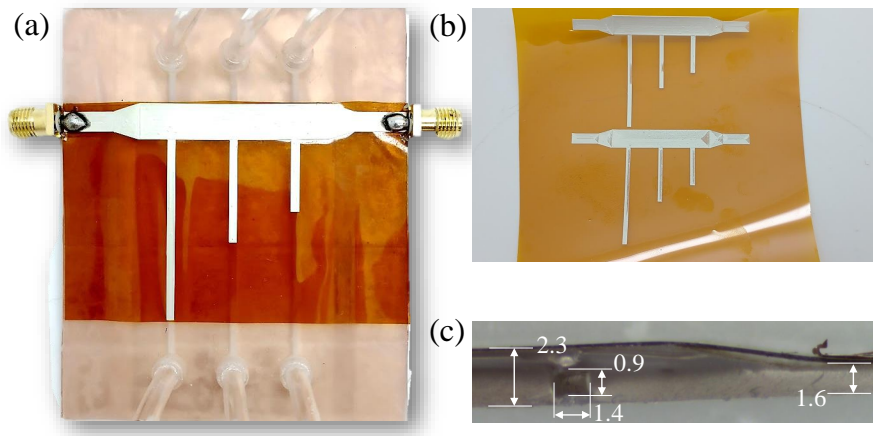

Fig. 8. Photographs of the fabricated sensor: (a) the assembled sensor; (b) the printed microstrip trace prior to adhesion; (c) cross-sectional micrograph of the substrate (dimensionsin $\mathrm{mm}$ ).

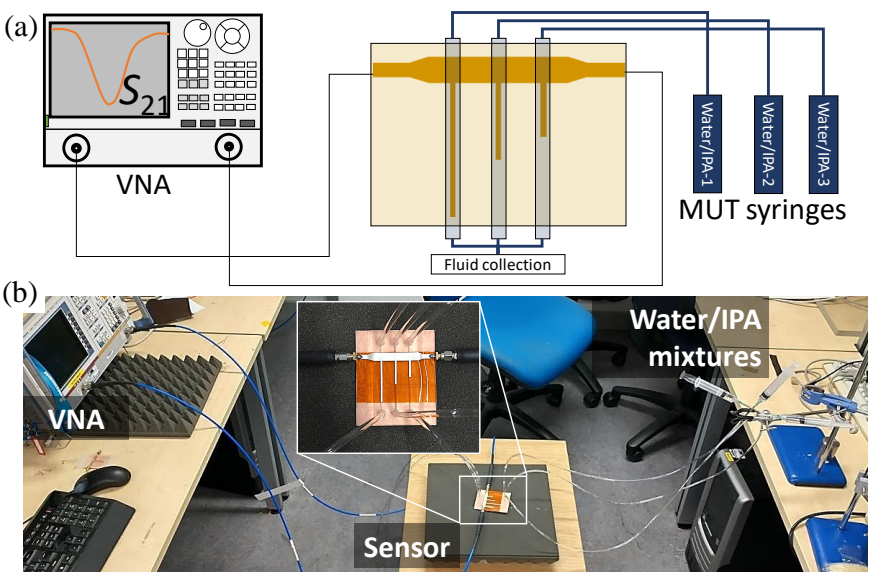

Fig. 9. Layout (a) and photograph (b) of the experimental setup.

of the fabricated sensor are shown in Fig. 8 .

Unlike previous works [14], [16], the proposed fabrication process is scalable and can be adjusted in real-time to control the microstrip line's dimensions based on the substrate's thickness. This overcomes using a manually-assembled copper tape ground plane as in [16], or an adhered copper film as in [14]. Therefore, the proposed fabrication method is a comparable lower cost alternative to standardized inkjet printing of microwave microfluidic sensors [?], [35].

\section{B. Sensor Measurements and Characterization}

The fabricated prototypes were measured using a two-port ZVB4 vector network analyzer (VNA), calibrated using a standard TOSM calibration. The 3D printed channels have been connected via long tubes, as shown in Fig. 9, to external syringes containing the MUT. The binary De-ionized (DI) water and IPA mixtures have been prepared by volume and kept in separate syringes. The measurements were carried out at a fixed room temperature to ensure the MUT's permittivity temperature-dependence does not influence the accuracy of the results.

The simulated and measured forward transmission of the bare sensor, i.e. with empty channels, are shown in Fig. 10. As the 3D CST model is based on the measured thickness of the fabricated sensor, based on the micrograph in Fig. 8(c),

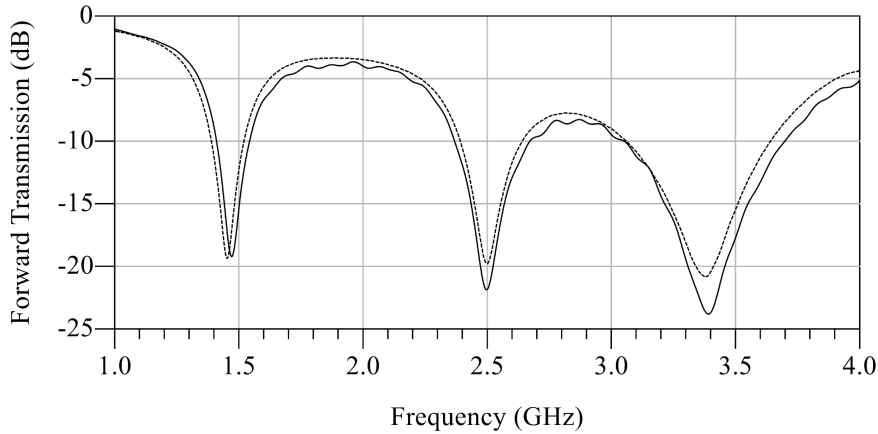

Fig. 10. Simulated (dashed) and measured (solid) $S_{21}$ of the unloaded sensor.

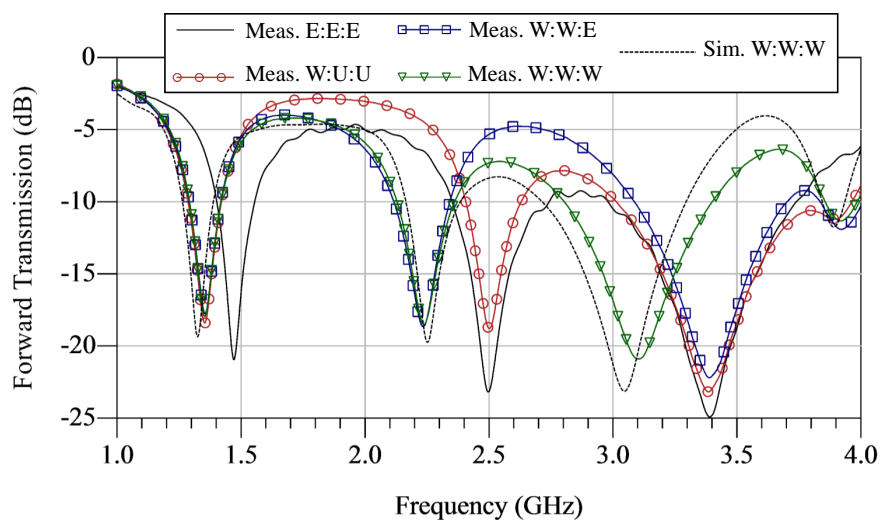

Fig. 11. $S_{21}$ of the sensor with DI water loading in different channels.

and the measured dielectric properties of the substrate, the observed close agreement is anticipated. This further demonstrates the advantage of using direct-write printing to realize the microstrip line, whose dimensions can be tuned after the microfluidic channel substrate is printed and characterized mechanically and electrically.

The channels have then been sequentially filled with DI water, to observe their loaded response. The water-loaded response can then be used as the calibration of the sensor, prior to characterizing different fluid samples [16]. Fig. 11 shows the simulated and measured $S_{21}$ response for DI water loading in different channels. From the sequential channel-loading, it can be seen that injecting water in a certain channel introduces a minimal influence on $f_{r}$ of the neighbouring channels. Along with the three fundamental resonant frequencies, around 1.45, 2.5 and $3.4 \mathrm{GHz}$, the higher-order resonance of the longest stub is observed around $3.9 \mathrm{GHz}$ in Fig. 11, but only when channel 1 is filled with water. This indicates that all three stubs are indeed within the bandwidth limits, previously discussed in Section II-B.

\section{Permittivity Measurements and Sensor EVALUATION}

\section{A. Permittivity Extraction and Accuracy Evaluation}

The next step in evaluating the sensor is to characterize its accuracy in measuring the real permittivity of an MUT. The formula linking $\Delta f_{r}$ to $\Delta \epsilon_{r}$ has been extracted using empirical fitting of the CST-simulated response of the sensor. 

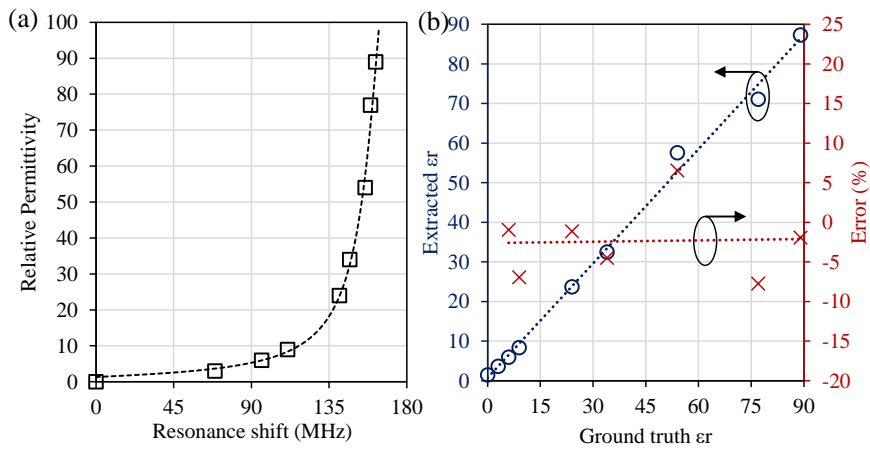

Fig. 12. Simulated (discrete points) and curve-fitted (continuous line) $\epsilon_{r} / f_{r}$ relationship (a) and accuracy (b), for channel 1.
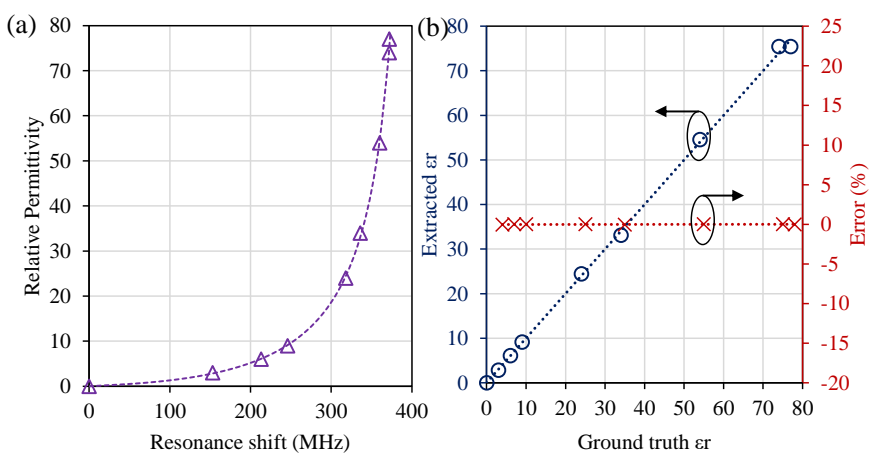

Fig. 13. Simulated (discrete points) and curve-fitted (continuous line) $\epsilon_{r} / f_{r}$ relationship (a) and accuracy (b), for channel 3.

As the simulations and measurements were found to be in good agreement, as observed in Fig. 10 and 11, the measured response is expected to be in agreement with the full-wave simulation. A $\Re\left\{\epsilon_{r}\right\}$ sweep from 1 to 90 was simulated. Fig. 12 and 13 show the $\epsilon_{r}$ and $\Delta f_{r}$ relation for channels 1 and 3 , respectively.

For channel 1, the relation observed in Fig. 12(a) has been fitted empirically using Matlab Curve-fitting toolbox. For channel $1, \epsilon_{r}$ is given by

$$
\epsilon_{1}=n \times \frac{m_{1} \Delta f_{1}^{2}+m_{2} \Delta f_{1}^{1.675}+m_{3} \Delta f_{1}+m_{4}}{m_{5} \Delta f_{1}^{2}+m_{6} \Delta f_{1}+m_{7}}
$$

where $m_{1}-m_{7}$ were obtained using curve fitting as: $m_{1}=16.35$; $m_{2}=-17.55 ; m_{3}=1.764 ; m_{4}=0.2324 ; m_{5}=6.048 ; m_{6}=-2.075$; $m_{7}=0.1778$, resulting in a high $R^{2}=0.995$.

In Fig. 12(a), it can be observed that the fitted values (dashed line) agree closely with the CST-simulated resonances (discrete points). $n$ in (5) is a tuning variable to be used after filling the channels with DI water, to calibrate the sensor's response after filling all the neighbouring channels with the MUT. In Fig. 12(b), it can be observed that the fitted relation exhibits a very high accuracy in evaluating $\epsilon_{r}$ from $f_{r}$.

The same approach was repeated for the other channels, for channels 2 and 3 , the relation is given by

$$
\epsilon_{3}=p \times \frac{q_{1} \Delta f_{3}^{2}+q_{2} \Delta f_{3}^{1.5}+q_{3} \Delta f_{3}+q_{4}}{q_{5} \Delta f_{3}^{2}+q_{6} \Delta f_{4}+q_{7}},
$$

where $q_{1}-q_{7}$ were evaluated using the same curve-fitting approach based on the simulated values in Fig. 13(a)

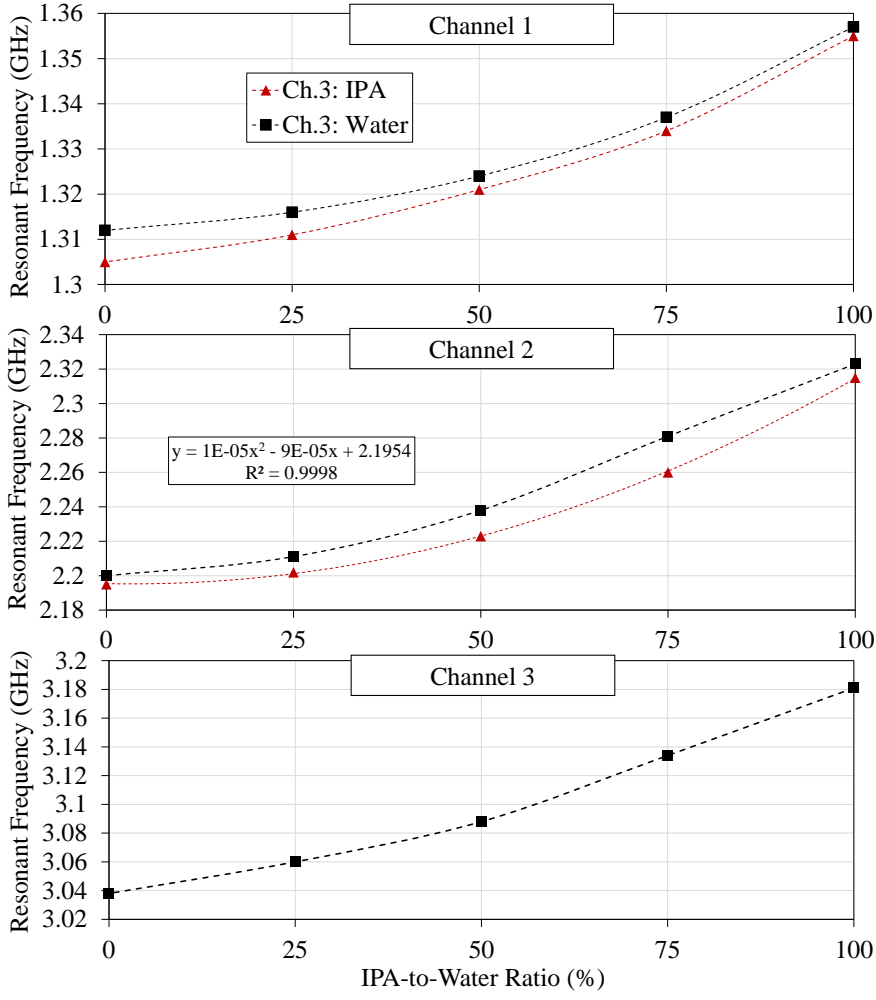

Fig. 14. Measured resonant frequency of the sensor's channels for binary DI Water/IPA mixtures, with water and IPA in the neighbouring channels.

to be: $q_{1}=10 ; q_{2}=-3.868 ; q_{3}=1.083 ; q_{4}=0 ; q_{5}=-0.3845$; $q_{6}=-0.01325 ; q_{7}=0.07019$, for channel 3. As observed in Fig. 13(a) and (b), the very high curve-fitting $R^{2}=0.9992$ results in a near-zero error in evaluating $\Re\left\{\epsilon_{r}\right\}$ from $\Delta f_{r}$.

Prior to filling the channels with the MUT, all channels were filled with DI water for calibration, and to evaluate the tuning parameters $n$ and $p$. Binary mixtures of IPA and water, at $100 \%, 75 \%, 50 \%, 25 \%$ and $0 \%$ IPA-to-water volume ratios were placed in each of the channels and $f_{r}$ was measured using the VNA. Fig. 14 shows the variation in $f_{r}$ for across the three channels, showing a very similar frequency-shift across all channels in response to the the variation in $\epsilon_{r}$ of the different IPA/water ratios.

Using a simple polynomial curve fitting, it can be observed that the measured results are highly linear with $R^{2}>0.999$. The measured $\Delta f_{r}$ for DI water-loading was used to obtain the tuning parameters $n, p$, and $r$, for channels 1,3 , and 2 , respectively. The fitted equations obtained from the $3 \mathrm{D}$ simulations were then used to evaluate the permittivity of the liquids based on the measured results, and the tuning parameters given by $n=1.11, p=1.55$, and $r=0.542$, for channels 1, 3, and 2, respectively. Fig. 15 shows the RFmeasured $\Re\left\{\epsilon_{r}\right\}$ of the binary mixtures, as evaluated by proposed the microwave microfluidic sensor.

In order to assess the accuracy of the measured values, the coaxial probe-measured values for binary water/IPA mixtures from [14] were used. The Debye model was then used to calculate $\epsilon_{r}$ for the frequencies of interest [36]. Fig. 15 shows the error percentage in the measured $\epsilon_{r}$ compared to the coaxial-probe measurements in [14], fitted using the Debye 


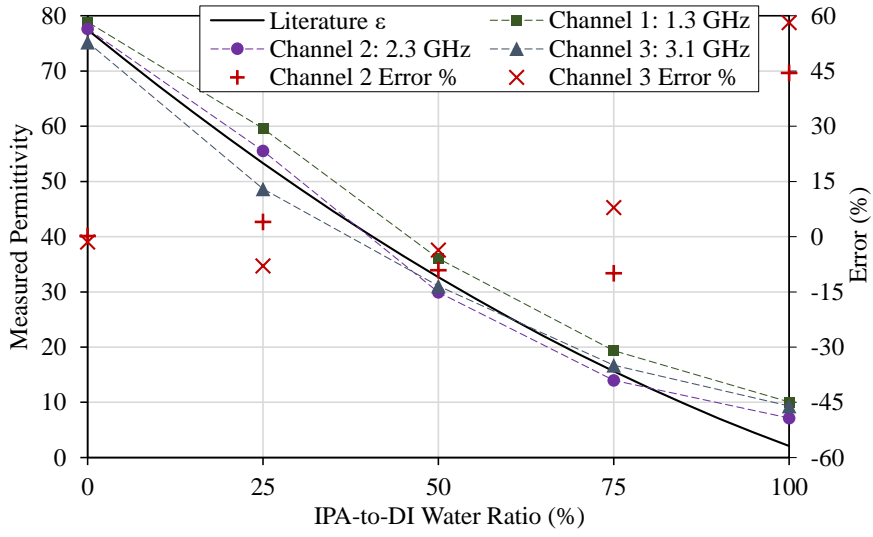

Fig. 15. Retrieved $\epsilon_{r}$ from the sensor's response and the error percentage of the proposed sensor.

curve for the three frequencies of operation [36].

Apart from the 100\%-IPA case, it can be observed that the sensor achieves at least $90 \%$ accuracy, which increases as the MUT approaches the permittivity of water. This is attributed to the utliized DI water-based calibration, which improves the sensor's accuracy for $\epsilon_{r}>20$. The maximum error of the proposed sensor is line with recently reported 3D-printed sensors [14], [22], which can only characterize a single MUT, compared in more detail in Section IV-C.

\section{B. Microfluidic Temperature Sensing}

As the permittivity of fluids is highly dependent on temperature, the proposed microfluidic sensor could be utilized to observe temperature variations through $\Delta f_{r}$. In this case, the MUT, e.g. water, will act as the temperature sensing element. Therefore, the temperature sensitivity could be improved by using an MUT with a high temperature $\epsilon_{r}$-dependence. As the previous experiments were carried out at room temperature, the 3D printed sensor was placed on a hot-plate as shown in Fig. 16(a). Due to the heat dissipation around the sensor, the temperature of the DI water sample inside the channel will be different from that of the hot plate's surface. Therefore, differential temperature measurements were take, where $\Delta f_{r}$ is characterized relative to the hot-plate's $\Delta T$.

The temperature has been varied from 60 to $85^{\circ} \mathrm{C}$. The temperature variation $\Delta T$ has been increased in $5^{\circ} \mathrm{C}$ increments. As observed across the three channels, in Fig. 16(b)-(d), all stubs exhibited a linear relation between temperature and $f_{r}$, showing the suitability of the sensor. Across all three channels, the sensor exhibits a varying sensitivty ranging from $\Delta f_{r} 2.4$ to $5 \mathrm{MHz} /{ }^{\circ} \mathrm{C}$. Compared to a recent microwave microfluidic temperature sensor, realized for evaluating the temperature of a single MUT [37], the proposed sensor, with a water-filled channel, achieves over 10-fold improvement in the sensitivity to temperature, from approximately $0.25 \mathrm{MHz} /{ }^{\circ} \mathrm{C}$ in [37], to $2.4-5 \mathrm{MHz} /{ }^{\circ} \mathrm{C}$. This highlights the benefits of utilizing a parallel-plate-based sensor with a high sensitivity, where the MUT's $\epsilon_{r}$ temperature-dependence could be used in a high-sensitivity readout circuit based on the proposed sensor structure.
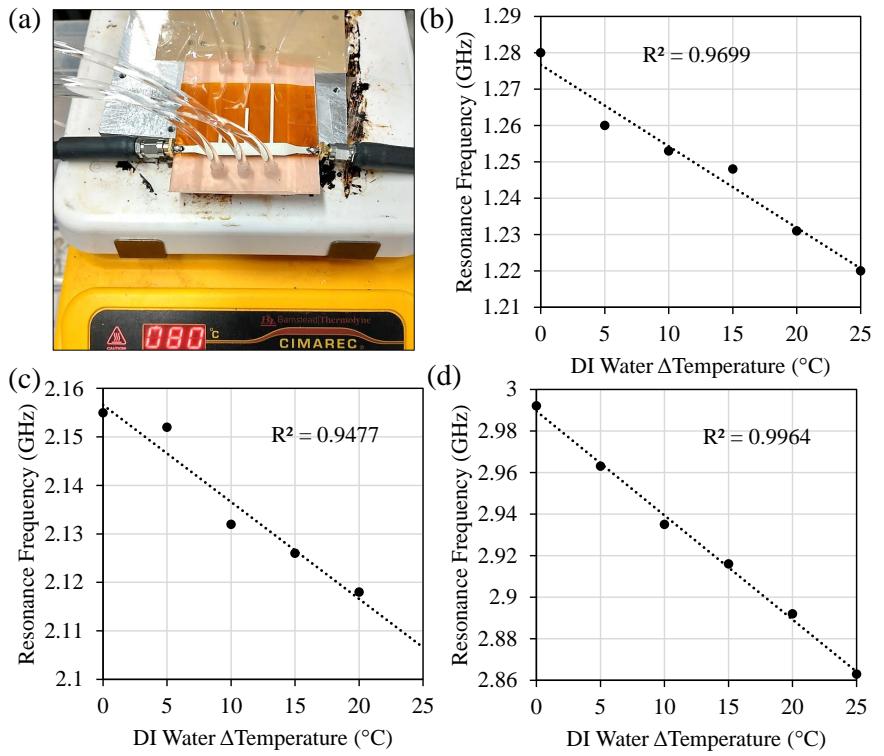

Fig. 16. Measured resonant frequency of the sensor's three channels filled with DI water as a function of the change in temperature: (a) measurement setup; (b) channel 1; (c) channel 2; (d) channel 3.

The temperature was not raised beyond $90^{\circ} \mathrm{C}$ due to the rated temperature of the cables $\left(85^{\circ} \mathrm{C}\right)$ and the glass-transition temperature of the SLA substrate. Nevertheless, in a realworld temperature sensing application, commercially-available high-temperature 3D-printed resins such as Roboze EXTEM could enable 3D-printed substrates to retain their properties as high as $247^{\circ} \mathrm{C}$. Moreover, using a read-out circuitry integrated on the same substrate, the need for external cabling and connectors is eliminated further improving the reliability and accuracy of the sensor, while reducing the test cost and time.

\section{Discussion and Comparison with Recent Sensors}

Observing the sensor's accuracy in characterizing the relative permittivity of binary fluid mixtures across all channels, the proposed design can be applied to a range of applications where a single-connection/single-instrument setup could be used to characterize multiple fluids. Therefore, while singleconnection microfluidic sensor calibration methods were proposed [33], there has been no complementary sensor design to-date, with the ability to characterize more than two materials with a single connection and read-out circuit.

Table I compares the fabricated prototype to recently reported microwave microfluidic sensors, either utilizing additive manufacturing or capable of sensing multiple fluids. While dual-mode resonators were proposed in [38] and [39], the proposed sensor is the only scalable multi-fluid microwave sensor proposed in literature. In addition to being the only reported sensor with $>2$ microfluidic channels and resonant elements, the proposed sensor's accuracy and sensitivity is comparable to state-of-the-art sensors which utilize additive manufacturing. Moreover, the proposed sensor maintains the lowest complexity in its fabrication process, which can also scale in volume, similaro to inkjet printed microfluidics [35]. To explain, while several sensors utilized 3D-printed channels 
TABLE I

COMPARISON WITH RECENT SIMILAR IMPLEMENTATIONS

\begin{tabular}{|l|l|l|l|l|}
\hline & \# MUTs & Water $S$ & Accuracy & Fabrication \\
\hline This work & 3 & $\begin{array}{l}0.10- \\
0.13 \%\end{array}$ & $92-99 \%$ & $\begin{array}{l}\text { 3D } \\
\text { dispenser } \\
\text { printing }\end{array}$ \\
\hline$[38]$ & 2 & $0.29 \%$ & Accuracy & $\begin{array}{l}\text { LTCC } \\
\text { milling }\end{array}$ \\
\hline$[39]$ & 2 & $0.22 \%$ & $98-99 \%$ & RF PCB \\
\hline$[14]$ & 1 & $0.15 \%$ & $81-99 \%$ & 3D-print. \\
\hline$[15]$ & 1 & $0.112 \%$ & $\begin{array}{l}\text { NR, } \\
99 \%^{\dagger}\end{array}$ & $\begin{array}{l}\text { 3D-print; } \\
\text { RF PCB }\end{array}$ \\
\hline$[22]$ & 1 & NA $^{*}$ & $90-99 \%$ & $\begin{array}{l}\text { 3D-print; } \\
\text { RF PCB. }\end{array}$ \\
\hline$[41]$ & 1 & $0.03 \%$ & $\approx 90 \%{ }^{\dagger}$ & RF PCB. \\
\hline
\end{tabular}

${ }^{*}$ Broadband non-resonant sensor; ${ }^{\dagger}$ estimated from the graph

[14], their integration was either done manually using copper tape [14], unsuitable for scalable manufacturing [16], or with complex high-frequency PCB on an expensive substrate [22]. On the other hand, the direct-written printed microstrip line could be easily adjusted post-fabrication to account for tolerances in the microfluidic channel. Moreover, the simple open-ended stub design, combined with direct-write printing, enable the operation frequency of the sensor to be adapted in a simple and scalable manner, without requiring a substrate re-design as in [14]. Moreover, while a four-band sensor was proposed based on multiple complimentary SRRs [40], the sensor was only used for characterizing a single MUT.

\section{CONCLUSION}

In this paper, a scalable microwave microfluidic sensor design was proposed for characterizing the real relative permittivity of multiple fluid samples. The simple design utilizing the parallel-plate capacitance of microstrip stubs enables simple analytical and numerical analysis, and is enabled by hybrid low-cost additive manufacturing. The proposed sensor has been experimentally characterized based on binary water/IPA mixtures showing at least $90 \%$ accuracy in determining the real-permittivity across all channels. The accuracy and sensitivity of the proposed sensor are in line with state-ofthe-art sensors while significantly improving the throughput and testing speed, owing to the utilization of three different channels. The proposed sensor was also demonstrated as a microfluidic temperature sensor exhibiting a high linearity and better sensitivity compared to similar microfluidic temperature sensors. The proposed design is expected to enable future lowcost fast-turnaround microfluidic sensors owing to its highly simplified microstrip structure, and due to utilizing low-cost hybrid 3D and dispenser printing.

\section{REFERENCES}

[1] P. Mezzanotte, V. Palazzi, F. Alimenti, and L. Roselli, "Innovative rfid sensors for internet of things applications," IEEE Journal of Microwaves, vol. 1, no. 1, pp. 55-65, 2021.

[2] C. Ramella, M. Pirola, and S. Corbellini, "Accurate characterization of high- $q$ microwave resonances for metrology applications," IEEE Journal of Microwaves, vol. 1, no. 2, pp. 610-624, 2021.
[3] B. S. Cook, J. R. Cooper, and M. M. Tentzeris, "An inkjet-printed microfluidic rfid-enabled platform for wireless lab-on-chip applications," IEEE Trans. Microw. Theory Techniq., vol. 61, no. 12, pp. 4714-4723, 2013.

[4] S. Liu, N. D. Orloff, C. A. E. Little, W. Zhao, J. C. Booth, D. F Williams, I. Ocket, D. M. M. . Schreurs, and B. Nauwelaers, "Hybrid characterization of nanolitre dielectric fluids in a single microfluidic channel up to 110 ghz," IEEE Trans. Microw. Theory Techniq., vol. 65 , no. 12 , pp. 5063-5073, 2017.

[5] R. Zhang, Q. Chen, K. Liu, Z. Chen, K. Li, X. Zhang, J. Xu, and E. Pickwell-MacPherson, "Terahertz microfluidic metamaterial biosensor for sensitive detection of small-volume liquid samples," IEEE Transactions on Terahertz Science and Technology, vol. 9, no. 2, pp. 209-214, 2019.

[6] R. Narang, S. Mohammadi, M. M. Ashani, H. Sadabadi, H. Hejazi, M. H. Zarifi, and A. Sanati-Nezhad, "Sensitive, Real-time and NonIntrusive Detection of Concentration and Growth of Pathogenic Bacteria using Microfluidic-Microwave Ring Resonator Biosensor," Sci Rep, vol. 8, p. 15807, 2018.

[7] G. Govind and M. J. Akhtar, "Metamaterial-inspired microwave microfluidic sensor for glucose monitoring in aqueous solutions," IEEE Sensors Journal, vol. 19, no. 24, pp. 11900-11907, 2019.

[8] L. Yang, R. Zhang, D. Staiculescu, C. P. Wong, and M. M. Tentzeris, "A Novel Conformal RFID-Enabled Module Utilizing Inkjet-Printed Antennas and Carbon Nanotubes for Gas-Detection Applications," IEEE Antennas Wireless Propag. Lett., vol. 8, pp. 653 - 656, 2009.

[9] X. Bao, M. Zhang, I. Ocket, J. Bao, D. Kil, Z. Liu, R. Puers, D. Schreurs, and B. Nauwelaers, "Integration of interdigitated electrodes in splitring resonator for detecting liquid mixtures," IEEE Transactions on Microwave Theory and Techniques, vol. 68, no. 6, pp. 2080-2089, 2020.

[10] P. Vélez, L. Su, K. Grenier, J. Mata-Contreras, D. Dubuc, and F. Martín, "Microwave microfluidic sensor based on a microstrip splitter/combiner configuration and split ring resonators (srrs) for dielectric characterization of liquids," IEEE Sensors Journal, vol. 17, no. 20, pp. 6589-6598, 2017.

[11] C. Lee and C. Yang, "Single-compound complementary split-ring resonator for simultaneously measuring the permittivity and thickness of dual-layer dielectric materials," IEEE Transactions on Microwave Theory and Techniques, vol. 63, no. 6, pp. 2010-2023, 2015.

[12] M. Schueler, C. Mandel, M. Puentes, and R. Jakoby, "Metamaterial inspired microwave sensors," IEEE Microwave Magazine, vol. 13, no. 2, pp. 57-68, 2012.

[13] N. Pandit, R. K. Jaiswal, and N. P. Pathak, "Plasmonic metamaterialbased label-free microfluidic microwave sensor for aqueous biological applications," IEEE J. Sens., vol. 20, no. 18, pp. 10 582-10 590, 2020.

[14] G. M. Rocco, M. Bozzi, D. Schreurs, L. Perregrini, S. Marconi, G. Alaimo, and F. Auricchio, "3-D Printed Microfluidic Sensor in SIW Technology for Liquids' Characterization," IEEE Trans. Microw. Theory Techn., vol. 68 no. 3, pp. 1175 - 1184, 2020.

[15] B. D. Wiltshire and M. H. Zarifi, "3-d printing microfluidic channels with embedded planar microwave resonators for rfid and liquid detection," IEEE Microwave and Wireless Components Letters, vol. 29, no. 1, pp. 65-67, 2019.

[16] A. Ebrahimi, J. Scott, and K. Ghorbani, "Ultrahigh-sensitivity microwave sensor for microfluidic complex permittivity measurement," IEEE Trans. Microw. Theory Techniq., vol. 67, no. 10, pp. 4269-4277, 2019.

[17] C. Mariotti, F. Alimenti, L. Roselli, and M. M. Tentzeris, "Highperformance rf devices and components on flexible cellulose substrate by vertically integrated additive manufacturing technologies," IEEE Transactions on Microwave Theory and Techniques, vol. 65, no. 1, pp. 62-71, 2017.

[18] V. Palazzi, P. Mezzanotte, F. Alimenti, M. Tentzeris, and L. Roselli, "Microfluidics-based 3d-printed $4 \times 4$ butler matrix in coaxial technology for applications up to k band," in 2019 IEEE MTT-S International Microwave Symposium (IMS), 2019, pp. 1371-1374.

[19] V. Palazzi, A. Cicioni, F. Alimenti, P. Mezzanotte, M. M. Tentzeris, and L. Roselli, "Compact 3-d-printed $4 \times 4$ butler matrix based on low-cost and curing-free additive manufacturing," IEEE Microwave and Wireless Components Letters, vol. 31, no. 2, pp. 125-128, 2021.

[20] M. Wagih, A. S. Weddell, and S. Beeby, "Millimeter-Wave Power Harvesting: A Review," IEEE Open Journal of Antennas and Propagation, vol. 1, pp. $560-578,2020$.

[21] S. Mohammadi, A. V. Nadaraja, D. J. Roberts, and M. H. Zarifi, "Real-time and hazard-free water quality monitoring based on microwave planar resonator sensor," Sensors and Actuators 
A: Physical, vol. 303, p. 111663, 2020. [Online]. Available: https://www.sciencedirect.com/science/article/pii/S0924424719307976

[22] J. Sorocki, I. Piekarz, K. Wincza, S. Gruszczynski, and J. Papapolymerou, "Broadband microwave microfluidic coupled-line sensor with 3-d-printed channel for industrial applications," IEEE Trans. Microw. Theory Techniq., vol. 68, no. 7, pp. 2808-2822, 2020.

[23] M. Wagih, A. S. Weddell, and S. Beeby, "Meshed High-Impedance Matching Network-Free Rectenna Optimized for Additive Manufacturing," IEEE Open Journal of Antennas and Propagation, vol. 1, pp. 615 $-626,2020$.

[24] J. Naqui, C. Damm, A. Wiens, R. Jakoby, L. Su, J. Mata-Contreras, and F. Martín, "Transmission lines loaded with pairs of stepped impedance resonators: Modeling and application to differential permittivity measurements," IEEE Transactions on Microwave Theory and Techniques, vol. 64, no. 11, pp. 3864-3877, 2016.

[25] O. Siddiqui, R. Ramzan, M. Amin, and O. M. Ramahi, "A NonInvasive Phase Sensor for Permittivity and Moisture Estimation Based on Anomalous Dispersion," Sci Rep, vol. 6, p. 28626, 2016.

[26] B. Wiltshire, K. Mirshahidi, K. Golovin, and M. H. Zarifi, "Robust and sensitive frost and ice detection via planar microwave resonator sensor," Sensors and Actuators B: Chemical, vol. 301, p. 126881, 2019.

[27] W. Ali and S. Al-Charchafchi, "Using equivalent dielectric constant to simplify the analysis of patch microstrip antenna with multi-layer substrates," in IEEE Antennas and Propagation Society International Symposium. 1998 Digest. Antennas: Gateways to the Global Network. Held in conjunction with: USNC/URSI National Radio Science Meeting (Cat. No.98CH36, vol. 2, 1998, pp. 676-679 vol.2.

[28] L. C. Fan, W. S. Zhao, D. W. Wang, Q. Liu, S. Chen, and G. Wang, "An ultrahigh sensitivity microwave sensor for microfluidic applications," IEEE Microwave and Wireless Components Letters, vol. 30, no. 12, pp. 1201-1204, 2020.

[29] G. M. Rocco, P. Barmuta, X. Bao, D. Schreurs, and M. Bozzi, "Efficient approach for dielectric permittivity measurements of liquids adopting a 3D-printed cavity resonator," Microw. Optical Technology Lett., p. Early View, 2021.

[30] A. B. Kouki, A. Khebir, R. G. Bosisio, and F. M. Ghannouchi, "A novel technique for the analysis of dielectric height variations in microstrip circuits," IEEE Transactions on Microwave Theory and Techniques, vol. 42, no. 1, pp. 73-77, 1994.

[31] A. H. Hamade, A. B. Kouki, and F. M. Ghannouchi, "A cad-suitable approach for the analysis of nonuniform mmic and mhmic transmission lines," IEEE Transactions on Microwave Theory and Techniques, vol. 44, no. 9, pp. 1614-1617, 1996.

[32] T.-H. Lin, S. N. Daskalakis, A. Georgiadis, and M. M. Tentzeris, "Achieving fully autonomous system-on-package designs: An embedded-on-package $5 \mathrm{~g}$ energy harvester within $3 \mathrm{~d}$ printed multilayer flexible packaging structures," in 2019 IEEE MTT-S International Microwave Symposium (IMS), 2019.

[33] X. Ma, N. D. Orloff, C. A. E. Little, C. J. Long, I. E. Hanemann, S. Liu, J. Mateu, J. C. Booth, and J. C. M. Hwang, "A multistate singleconnection calibration for microwave microfluidics," IEEE Transactions on Microwave Theory and Techniques, vol. 66, no. 2, pp. 1099-1107, 2018.

[34] M. Wagih, "Direct-Write Dispenser Printing for Rapid Antenna Prototyping on Thin Flexible Substrates," in 2020 European Conference on Antennas and Propagation (EuCAP), 2020.

[35] "Fully inkjet-printed microfluidics: a solution to low-cost rapid threedimensional microfluidics fabrication with numerous electrical and sensing applications," Sci. Rep.

[36] "Dielectric relaxation spectroscopy of 2-propanol-water mixtures," $J$. Chem. Phys.

[37] "Microwave temperature measurement in microfluidic devices," Lab Chip.

[38] N. Jankovic and V. Radonic, "A microwave microfluidic sensor based on a dual-mode resonator for dual-sensing applications," Sensors, vol. 17, no. $12,2017$.

[39] S. Kiani, P. Rezaei, and M. Navaei, "Dual-sensing and dual-frequency microwave srr sensor for liquid samples permittivity detection," Measurement, vol. 160, p. 107805, 2020

[40] M. A. H. Ansari, A. K. Jha, Z. Akhter, and M. J. Akhtar, "Multi-band rf planar sensor using complementary split ring resonator for testing of dielectric materials," IEEE Sensors Journal, vol. 18, no. 16, pp. 65966606, 2018

[41] E. L. Chuma, Y. Iano, G. Fontgalland, and L. L. Bravo Roger, "Microwave sensor for liquid dielectric characterization based on metamaterial complementary split ring resonator,' IEEE Sensors Journal, vol. 18, no. 24 , pp. $9978-9983,2018$.

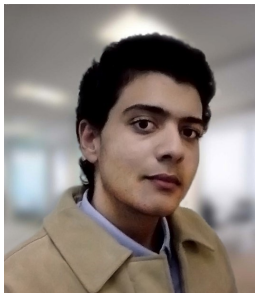

Mahmoud Wagih (GS'18, M'21) received his B.Eng. (Hons.) from the University of Southampton in September 2018, and his Ph.D. in April 2021.

In 2017 he worked as a Research Assistant at the University of Southampton. In 2018, he was a Hardware Engineering Intern at Arm, and, in 2020 a Research Intern at Arm, Cambridge, U.K. He is currently a Research Fellow at the University of Southampton, U.K., leading a Flexible Innovation Fund project on Green RF Sensing. His interests broadly cover antennas and microwave systems in energy harvesting, sensing, and wearable applications. He has over 40 refereed journal and conference publications, and has delivered several invited webinars on these topics.

Dr. Wagih is a member of the Institute of Engineering and Technology (MIET). He was the recipient of the Best Undergraduate Project Prize, School Winner Doctoral Research Award, Best in Faculty Doctoral Research Award, and the Dean's Award for Early Career Researchers, in 2018-2021, at the University of Southampton. He was selected for the IEEE International Microwave Symposium Project Connect in 2019. He received the Best Student Paper Award at the IEEE Wireless Power Transfer Conference, 2019, the Best Oral Paper at PowerMEMS, 2019, and was a Best Student Paper Finalist at IEEE WPTC, 2021. He won the MTT-S Best 3MT Presentation Prize (second place) at the IEEE Microwave Week, 2020, and was a session co-chair at the European Conference on Antennas and Propagation, 2021. He acts as a reviewer for nine TRANSACTIONS and journals. He is an affiliate member of the IEEE MTT-S RFID, Wireless Sensors and IoT Technical Committee (TC-26).

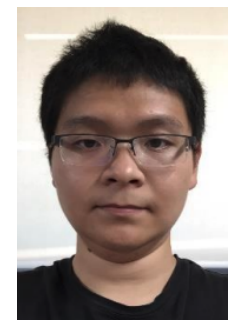

Junjie Shi received a B.Sc. degree from the Nanjing Institute of Technology, China in 2008, an M.Sc. degree in Microelectromechanical systems in 2012, and a Ph.D. in 2017 at the University of Southampton.

He was appointed as a Research Fellow in the School of Electronics and Computer Science (ECS) in 2017. His research interest covers a board range of wearables including but not limited to sensors, actuators, memory, and energy harvesting etc. As well as those wearable devices, he is interested in the fabrication technologies, such as 3D printing, dispenser printing, inkjet printing, micro-fabrications, which enable conventional and novel devices to be directly fabricated on to textiles. 